DOI: https://doi.org/10.17308/geology.2021.3/3653

Поступила в редакцию: 27.05.2021

Принята к публикации: 02.09.2021

Опубликована онлайн: 07.10.2021

\title{
Электрические зондирования с комбинированной и симметричной установками над геологическими неоднородностями: математическое моделирование и экспериментальные примеры
}

\author{
(С2019 О. И. Федорова ${ }^{\bowtie}$ \\ Институт геофизики УрО РАН, ул. Амундсена, д.100, \\ 620016, Екатеринбург, Российская Федерация
}

\begin{abstract}
Аннотация
Введение: При решении геологических и инженерно-геологических задач широко применяются электрические зондирования на постоянном токе с использованием четырехэлектродных симметричных установок AMNB - Шлюмберже $(S)$ и Веннера $(\mathrm{V})$, что позволяет для горизонтально-слоистых сред достаточно уверенно определять мощность и удельное сопротивление слоев. Геологические неоднородности, присутствующие в среде, создают краевые эффекты, отражающиеся на разрезах кажущихся сопротивлений дополнительными аномальными областями и влияющие на погрешность количественной интерпретации. Для уменьшения краевых эффектов была предложена методика электрических зондирований с комбинированной установкой $\mathrm{AMN+NMA} \mathrm{B \rightarrow \infty ,} \mathrm{в} \mathrm{кото-}$ рой проводятся двойные измерения относительно центрального приемного заземления (M).

Методика: Выполнено математическое моделирование зондирований с установками AMNB (S) и AMN+NMA над локальными объектами: шаром, полушаром, аппроксимирующими геоэлектрические неоднородности. Проведены экспериментальные исследования на двух объектах, используя комбинированную и, для сравнения, симметричную установки.

Результаты: По рассчитанным теоретическим разрезам кажущихся сопротивлений показано, что приповерхностные и погруженные локальные неоднородности четко оконтуриваются комбинированной установкой с минимальным проявлением краевых эффектов. На разрезах, полученных симметричной установкой, имеются краевые эффекты, и нижняя граница объекта плохо определяется. Экспериментальные примеры выявили различия в структуре разрезов кажущихся сопротивлений для рассматриваемых установок, которые зависят от геологических особенностей среды. Над средой с горизонтальной слоистостью разрезы близки по структуре. Результаты инверсии отличаются в основном разной вертикальной мощностью слоев. Над коренными породами с большим удельным сопротивлением, в приконтактных зонах кривые зондирования и результаты инверсии могут значительно отличаться.

Bblвoдbl: Исходя из проведенного моделирования и экспериментов, можно заключить, что комбинированная установка AMN+NMA имеет некоторые преимущества по сравнению с широко применяемой AMNB установкой и может использоваться для электрических зондирований верхней части геологического разреза, а также в электротомографии горных пород.

Ключевые слова: Электрические зондирования, комбинированная установка, симметричная установка, разрез кажущихся сопротивлений, геоэлектрическая неоднородность, краевые эффекты.

Для изитирования: Федорова О. И. Электрические зондирования с комбинированной и симметричной установками над геологическими неоднородностями: математическое моделирование и экспериментальные примеры // Вестник Воронежского государственного университета. Серия: Геология. 2021. №3. C. 87-95. DOI: https://doi.org/10.17308/geology.2021.3/3653
\end{abstract}

Контент доступен под лицензией Creative Commons Attribution 4.0 License.

Федорова Ольга Ивановна, e-mail: fougeo@mail.ru

Вестник Воронежского государственного университета. Серия: Геология. 2021, № 3, 87-95 


\section{Введение}

В геофизической практике для решения геологических и инженерно-геологических задач широко применяются электрические зондирования на постоянном токе. В основном зондирования выполняют с использованием четырехэлектродных симметричных установок AMNB - Шлюмберже (S) и Веннера (V), что позволяет для горизонтально-слоистых сред достаточно уверенно определять мощность и удельное сопротивление слоев. Верхняя часть земной коры наиболее подвержена интенсивным геологическим процессам, поэтому горизонтальная слоистость залегания горных пород, особенно на небольших глубинах, нарушена с образованием геологических неоднородностей. Приповерхностные неоднородности являются помехами, так как трансформируют кривую зондирования и вносят неверное представление о глубинном строении среды. На малых глубинах в электрическом поле от локальных геологических объектов могут возникать краевые эффекты, которые затрудняют определение размеров неоднородности при интерпретации. Краевые эффекты искажают общую структуру геоэлектричекого разреза. При зондировании с установкой Веннера несколько уменьшается влияние приповерхностных неоднородностей, находящихся вблизи приемной линии (MN), поскольку в процессе измерений одновременно увеличивается разнос питающей линии (AB) и размер приемной.

Методы сопротивлений совершенствуются в направлении разработок новых технологий. Наиболее популярный и хорошо себя зарекомендовавший - метод томографии электросопротивления среды, где чаще всего используются установки Шлюмберже и Венера с 2-D, 3-D инверсией [1-4]. В МГУ разработан метод сплошных электрических зондирований (СЭ3), в котором много общего с томографией. Отличительной особенностью СЭЗ является выделение анализа искажений от неоднородностей в отдельный этап с последующей 1-D и 2-D интерпретацией [5]. Хотя современные программы сужают рамки эквивалентности решения обратной задачи, но в сложных неоднородных средах добиться однозначности решения невозможно. В силу технических возможностей метод томографии применяется в основном при малоглубинных исследованиях.

Для уменьшения краевых эффектов от неоднородностей была предложена методика электрических зондирований с комбинированной установкой $\mathrm{AMN}+\mathrm{NMA} \mathrm{B} \rightarrow \infty$ (рис. 1, c), в которой применяется трехэлектродная установка Гуммеля AMN B $\rightarrow \infty$, в ней электроды находятся на одной прямой и $\mathrm{AM}=\mathrm{MN}$ [6]. Суть методики состоит в том, что на каждом разносе выполняются двойные измерения. Сначала одно крайнее заземление подключается к генератору тока, два других к приемнику, затем второе крайнее заземление к генератору, а первое к приемнику. Центральный электрод (М) всегда подключен к приемнику. Вычисляется среднее значение $\rho_{\kappa}$, которое относится к приемному электроду (М). Теоретические расчеты над проводящим шаром и физическое моделирование над проводящим параллелепипедом, помещенным в однородную среду, показали, что за счет двусторонних измерений относительно центрального приемного электрода зондированиями с установкой AMN+NMA на разрезе кажущихся сопротивлений четко оконтуривается неоднородность, и значительно меньше проявляются краевые эффекты, чем с установкой AMNB (S) [7]. В работе [8] выполнено математическое моделирование над вертикальным пластом, аппроксимирующим вертикально-слоистую среду. Контакт вмещающей среды с пластом лучше выделяется симметричной установкой AMNB (S), но на разрезах кажущихся сопротивлений присутствуют краевые эффекты. При зондированиях с комбинированной установкой краевые эффекты практически отсутствуют.

Электрические зондирования часто применяют при мелкомасштабном геокартировании, где измерения выполняются с небольшой дискретностью. В этом случае обработку данных можно проводить по программам 1D инверсии, которые разработаны для горизонтально-слоистой среды. Над такой средой результаты зондирований с комбинированной установкой $\mathrm{AMN}+\mathrm{NMA}$ и с установкой Гуммеля AMN одинаковы. Было выполнено математическое моделирование электрических зондирований над горизонтально-слоистой средой для установок Шлюмберже, Веннера и Гуммеля при одинаковом положении их крайних электродов (рис.1). Установка Гуммеля является частью установки Венера, поэтому теоретические кривые кажущихся сопротивлений с установкой Гуммеля представляют собой кривые от установки Веннера, смещённые по оси разносов на 1/3 к началу координат. Кривые зондирования с установкой Шлюмбереже и Веннера медленнее выходят на асимптоту последнего слоя по сравнению с установкой AMN из-за меньшей глубины проникновения тока. Таким образом, было показано, что для горизонтально-слоистой среды интерпретацию данных, полученных с комбинированной установкой, можно проводить по программам 1D инверсии для установки Веннера с разносом AB/3 [9].

(a)

(b)

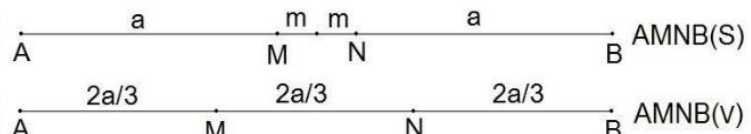

(c) $\mathrm{A}$

N

a

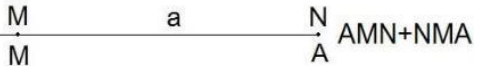

Pис. 1. Схема установок: a - AMNA (S); b - AMNB (V); cAMN+NMA.

[Fig. 1. The arrays: a - AMNA (S); b - AMNB (V); c $\mathrm{AMN}+\mathrm{NMA}]$

Экспериментальные исследования, проведенные зондированиями с установками AMNB (S) и $\mathrm{AMN+NMA,} \mathrm{выявили} \mathrm{различия} \mathrm{в} \mathrm{структуре} \mathrm{разрезов}$ кажущихся сопротивлений, особенно вблизи дневной поверхности в рыхлых осадочных породах. Поэтому возникла необходимость в дополнительных расчетах и натурных экспериментах. В статье рассматривается 
математическое моделирование для зондирований с комбинированной $\mathrm{AMN}+\mathrm{NMA}$ и симметричной AMNB (S) (вертикальные электрические зондирования - ВЭЗ) установками над полушаром и шаром. Приводятся результаты экспериментальных исследований.

\section{Математическое моделирование}

Теоретические расчеты выполнены по известным решениям электрического потенциала в поле точечного источника в присутствии неоднородности. Кажущееся сопротивление вычисляли по выражению:

$$
\rho_{k}=\frac{\left(U_{M}-U_{N}\right)}{I} k,
$$

где $\mathrm{U}_{\mathrm{M}}$ и $\mathrm{U}_{\mathrm{N}}$ - электрические потенциалы в приемных электродах; I - сила тока; $k$ - геометрический параметр. Для симметричной установки $\mathrm{AMNB}(\mathrm{S})$ :

$$
k=\frac{\pi\left(a^{2}-m^{2}\right)}{2 m},
$$

для комбинированной установки AMN+NMA (рис.1):

$$
k=4 \pi a .
$$

Разрезы построены от полуразноса $\mathrm{r}=\mathrm{AB} / 2$ и $\mathrm{r}=\mathrm{AM}$ для симметричной и комбинированной установок, соответственно. Для моделей шара и полушара все линейные размеры приведены к их радиусам.

Разрезы кажущихся сопротивлений над полушаром. Расчеты зондирований выполнены на дневной поверхности однородного полупространства в присутствии полушара, выходящего на поверхность, с использованием выражений для точечного источника, расположенного вне и внутри полушара [10]:

$$
\begin{gathered}
U_{1 \mathrm{~A}}=\frac{I \rho_{1}}{2 \pi}\left(\frac{1}{R}+\beta_{21} \sum_{n=0}^{\infty} \frac{n b^{2 n+1}}{\left(n+\alpha_{21}\right)\left(r_{0} r\right)^{n+1}} P_{n}(\cos \theta)\right), \\
U_{2 \mathrm{~A}}=\frac{I \rho_{1}}{2 \pi}\left(\frac{\alpha_{21}}{r_{0}} \sum_{n=0}^{\infty} \frac{2 n+1}{n+\alpha_{21}}\left(\frac{r}{r_{0}}\right)^{n} P_{n}(\cos \theta)\right),
\end{gathered}
$$

a

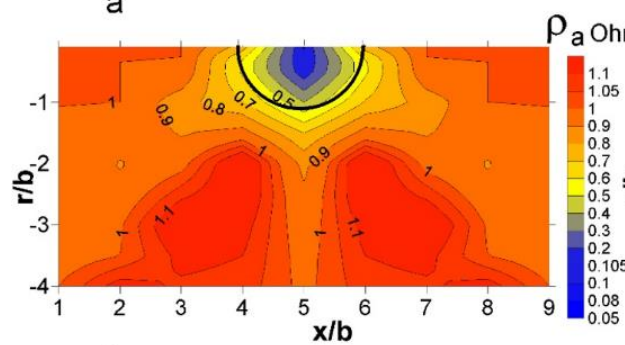

b

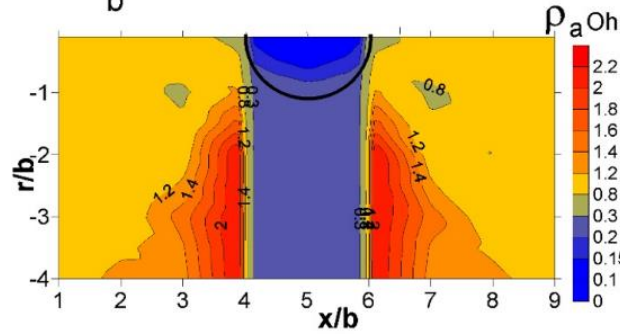

$$
\begin{gathered}
U_{1 \mathrm{~B}}=\frac{I \rho_{2}}{2 \pi}\left(\frac{\alpha_{12}}{r} \sum_{n=0}^{\infty} \frac{2 n+1}{n+\alpha_{21}}\left(\frac{r_{0}}{r}\right)^{n} P_{n}(\cos \theta)\right), \\
U_{2 \mathrm{~B}}=\frac{I \rho_{2}}{2 \pi}\left(\frac{1}{R}+\beta_{12} \sum_{n=0}^{\infty} \frac{n+1}{\left(n+\alpha_{21}\right)} \frac{\left(r r_{0}\right)^{n}}{b^{2 n+1}} P_{n}(\cos \theta)\right), \\
\text { где } \alpha_{\mathrm{ij}}=\frac{\rho_{i}}{\rho_{i}+\rho_{j}}, \beta_{i j}=\frac{\rho_{i}-\rho_{j}}{\rho_{i}+\rho_{j}},
\end{gathered}
$$

$\mathrm{U}_{1 \mathrm{~A}}$, и $\mathrm{U}_{2 \mathrm{~A}}$ - потенциал вне и внутри полушара, coответственно, когда токовый электрод находится вне полушара, $\mathrm{U}_{1 \mathrm{~B}}$, и $\mathrm{U}_{2 \mathrm{~B}}$ - потенциал вне и внутри полушара, соответственно, когда токовый электрод находится внутри полушара, $\rho_{1}$ и $\rho_{2}-$ удельные сопротивления вмещающей среды и полушара, $b$ - его радиус. Расстояние от измерительного и токового электродов до центра полушара $-r$ и $r_{0}$. Расстояние от токового и измерительного электрода $-\mathrm{R} . \mathrm{P}_{\mathrm{n}}(\cos \theta)=1$, когда измерительный и токовый электроды лежат по одну сторону от центра полушара, и $\mathrm{P}_{\mathrm{n}}(\cos \theta)=(-1)^{\mathrm{n}}$, когда по разные стороны.

Расчеты выполнили для непроводящего ( $\rho_{1}=0.1$ Ом $\cdot \mathrm{M}, \rho_{2}=1$ Ом·м) и проводящего $\left(\rho_{1}=1\right.$ Ом $\cdot \mathrm{M}, \rho_{2}=0.1$ Ом·м) полушара. На разрезах кажущихся сопротивлений полушар лучше выделяется комбинированной установкой (рис. 2 a, b). Проводящий полушар оконтуривается по изолинии 0.5-0.6, краевые эффекты проявляются незначительно. Аномалия от непроводящего полушара локализуется, не выходя за его границы, а краевые эффекты практически отсутствуют. При зондировании с симметричной установкой на разрезе ниже полушара образуется вытянутая на глубину ложная аномалия, затрудняющая определение размера локального объекта (рис. 2 c, d). В районе аномалии отмечаются краевые эффекты, увеличивая кажущееся сопротивление вмещающей среды более чем в два раза.

C

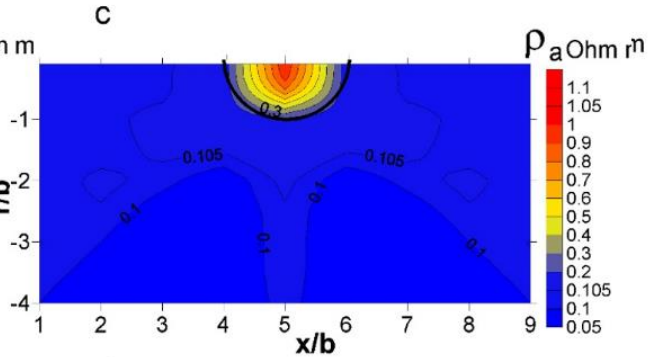

d

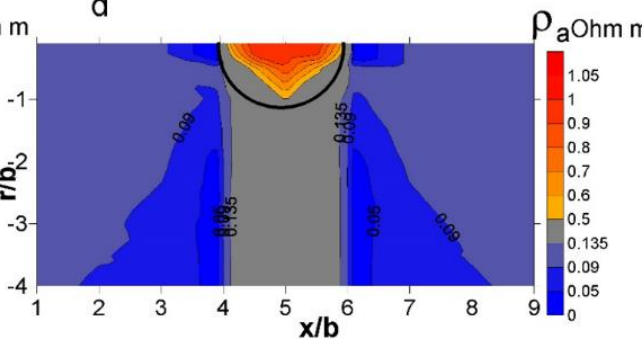

Рис. 2. Разрезы кажущихся сопротивлений над полушаром. Проводящий полушар $\rho_{2}=0.1$ Ом·м, $\rho_{1}=1$ Ом·м: $a-$ установка $\mathrm{AMN+NMA;} c$ - установка AMNB (S). Непроводящий полушар $\rho_{2}=1$ Ом·м, $\rho_{1}=0.1$ Ом·м: $b-$ установка AMN+NMA; $d-$ установка AMNB (S).

[Fig. 2. The sections of apparent resistivity over the hemisphere. Conductive hemisphere $\rho 2=0.1 \mathrm{Ohm} \cdot \mathrm{m}, \rho 1=1 \mathrm{Ohm} \cdot \mathrm{m}$ : (a) AMN+NMA array; (c) - AMNB (S) array. Non-conductive hemisphere $\rho 2=1 \mathrm{Ohm} \cdot \mathrm{m}, \rho 1=0.1 \mathrm{Ohm} \cdot \mathrm{m}$ : (b) - AMN+NMA array; (d) AMNB (S) array.] 
Разрезы кажущихся сопротивлений над шаром. Теоретические разрезы $\rho_{к}$ над шаром рассчитаны, с использованием известного выражения потенциала на дневной поверхности для погруженного шара в поле точечного электрического источника [11]:

$$
\begin{gathered}
U(\mathrm{r}, \theta)=\frac{I \rho_{1}}{2 \pi}\left[\frac{1}{R}+2 \sum_{n=0}^{\infty} p_{n} \frac{b^{2 n+1}}{\left(r r_{0}\right)^{n+1}} P_{n}(\cos \theta)\right], \\
\text { где } p_{n}=\frac{\left(\rho_{2}-\rho_{1}\right) n}{\rho_{1} n+\rho_{2}(n+1)},
\end{gathered}
$$

где $\rho_{1}$ и $\rho_{2}-$ удельные сопротивления вмещающей среды и шара соответственно, $b$ - его радиус, $r$ и $r_{0}-$ расстояние от измерительного и токового электродов до центра шара, $\mathrm{R}$ - расстояние от токового и измерительного электрода.
Расчетные параметры модели над шаром идентичны параметрам над полушаром. На разрезах кажущихся сопротивлений над проводящим и непроводящим шаром аномальная область имеет схожую структуру. При зондировании с комбинированной установкой верхняя граница шара четко оконтуривается. Нижняя граница для проводящего шара определяется изолинией 0.8 , для непроводящего - 0.13. Краевые эффекты здесь проявляются незначительно (рис. 3 a, b). Для симметричной установки нижняя граница шара с вмещающей средой смещена вглубь разреза, искажая вертикальные размеры объекта (рис. 3 b, d). Горизонтальные размеры локальной неоднородности определяются одинаково для обеих установок.

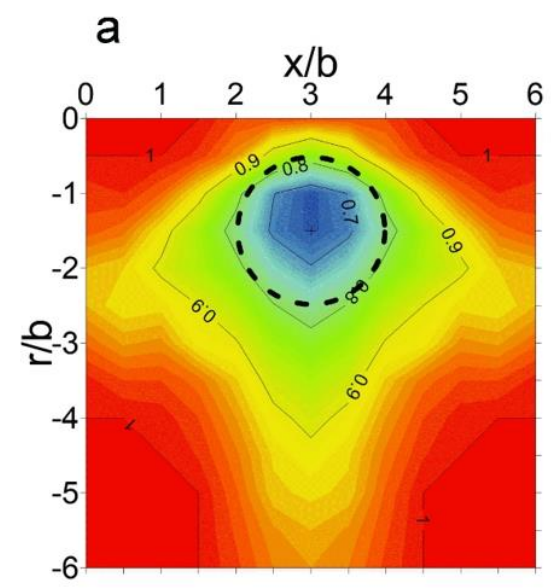

b

C

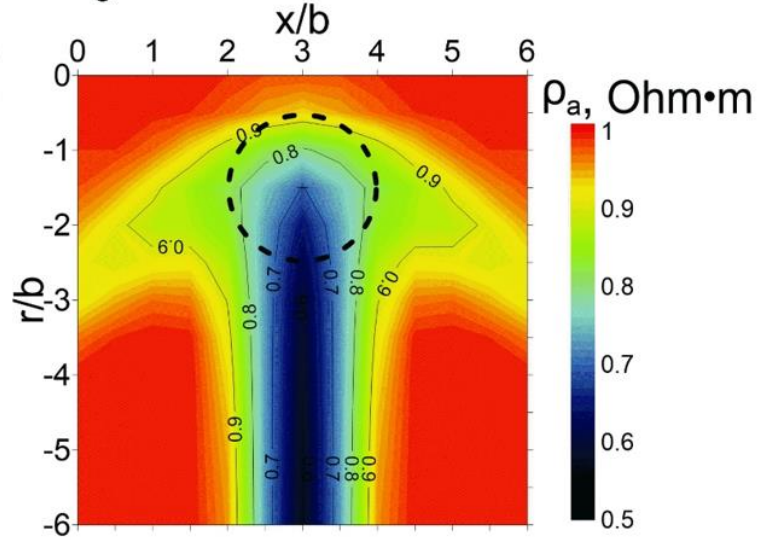

d
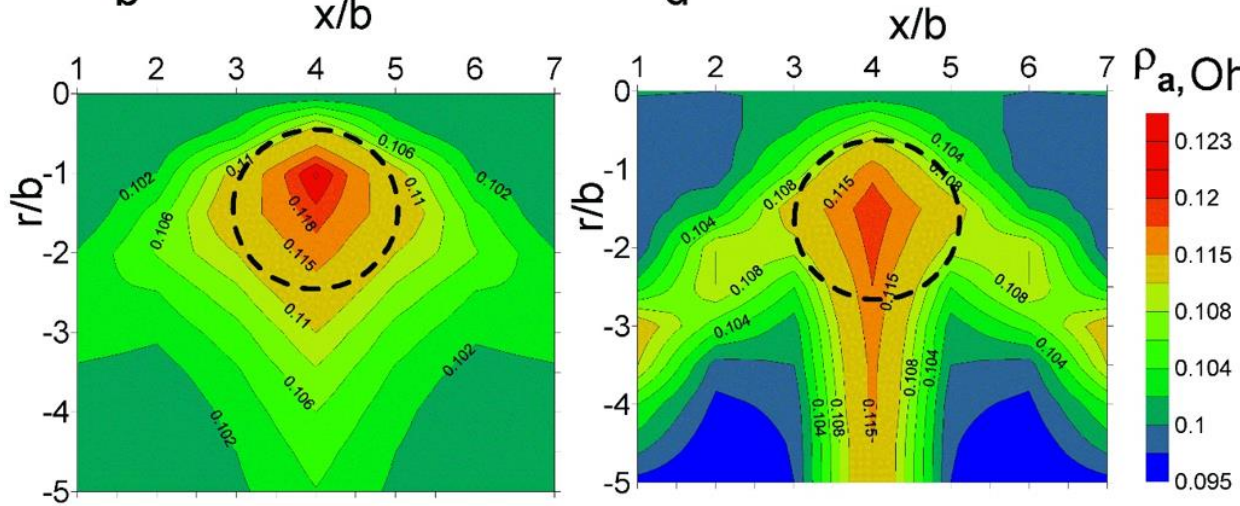

Рис. 3. Разрезы кажущихся сопротивлений над шаром. Проводящий шар $\rho_{2}=0.1$ Ом·м, $\rho_{1}=1$ Ом·м: $a-$ установка AMN+NMA; $c$ - установка AMNB (S). Непроводящий шар $\rho_{2}=1$ Ом·м, $\rho_{1}=0.1$ Ом·м: $b$ - установка AMN+NMA; $d$ - установка AMNB (S).

[Fig. 3. The sections of apparent resistivity over the sphere. Conductive sphere $\rho 2=0.1 \mathrm{Ohm} \cdot \mathrm{m}, \rho 1=1 \mathrm{Ohm} \cdot \mathrm{m}$ : (a) - AMN+NMA array; (c) $-\mathrm{AMNB}(\mathrm{S})$ array. Non-conductive sphere $\rho 2=1 \mathrm{Ohm} \cdot \mathrm{m}, \rho 1=0.1 \mathrm{Ohm} \cdot \mathrm{m}$ : (b) - AMN+NMA array; (d) - AMNB (S) array.]

\section{Результаты экспериментальных исследований}

Методика электрических зондирований с установкой AMN+NMA была опробована на двух объектах. В комплекс исследований включали вертикальные электрические зондирования с установкой AMNB (S), что позволило сравнить результаты двух методик. Схема измерительных установок приводится на рисунке 1. Крайние электроды подключались сначала к генератору в установке ВЭЗ (питающая линия АВ), затем выполнялись двойные измерения с установкой AMN+NMA. Исследования проводили аппаратурой
ЭРА-МАКС (НПП ЭРА, г.Санкт-Петербург). Кажущееся сопротивление для установки AMNB (S), вычисляли по известному выражению для вертикальных электрических зондирований. По измерениям, полученным с установкой AMN+NMA, значения кажущихся сопротивлений рассчитывали по формуле:

$$
\rho_{k}^{c p}=\left(\rho_{k}^{\mathrm{AMN}}+\rho_{k}^{\mathrm{NMA}}\right) / 2 .
$$

Интерпретацию проводили по программе IPI 2win (1-D инверсия) [12]. Для обработки данных, полученных комбинированной методикой, применяли вариант 
с установкой Веннера от разноса AB/3, предусмотренный программой.

Комплексные электрические зондирования провели при изучении состояния грунтовой плотины, ограждающей водоем для хранения жидких химических веществ. Плотина сложена из глинисто-щебенистого материала, имеет высоту в среднем 10 м. Подстилающие коренные породы представлены тремя типами: порфиритами, туфами и известняками. Широко развита трещиноватость скальных пород, а контакты туфов с порфиритами осложнены тектоническими нарушениями. Осадочные образования состоят из элювиальных и делювиальных отложений коры выветривания коренных пород, торфа и аллювия. Профиль наблюдений проходил по откосу плотины, который находится примерно на 5 м ниже от ее гребня. Откос имеет устойчивое состояние и сложен песчано-глинистым материалом. Точки наблюдений располагались через 20 м. Полуразносы питающей линии $(\mathrm{AB} / 2)$ для установки Шлюмбирже составляли: 1.5 ; $2,2.8 ; 3.8 ; 5 ; 7 ; 10 ; 15 ; 20$ м. Фактические разносы от питающего электрода (А) до центра приемной линии (MN) в комбинированной установке при одинаковом положении крайних электродов для обеих установок составляли: $2.25 ; 3 ; 4.2 ; 5.7 ; 7.5 ; 10.5 ; 15 ; 22.5 ; 30$ м, то есть в 1.5 раза больше разносов установки AMNB (S). Но поскольку в новой методике зондирований измерения относим к центральному неподвижному приемному электроду (M), за разнос приняли расстояние $\mathrm{AM}=\mathrm{AB} / 2$ (рис. 1).

a

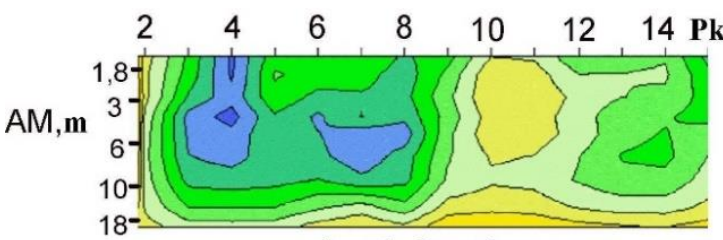

geoelectrical section
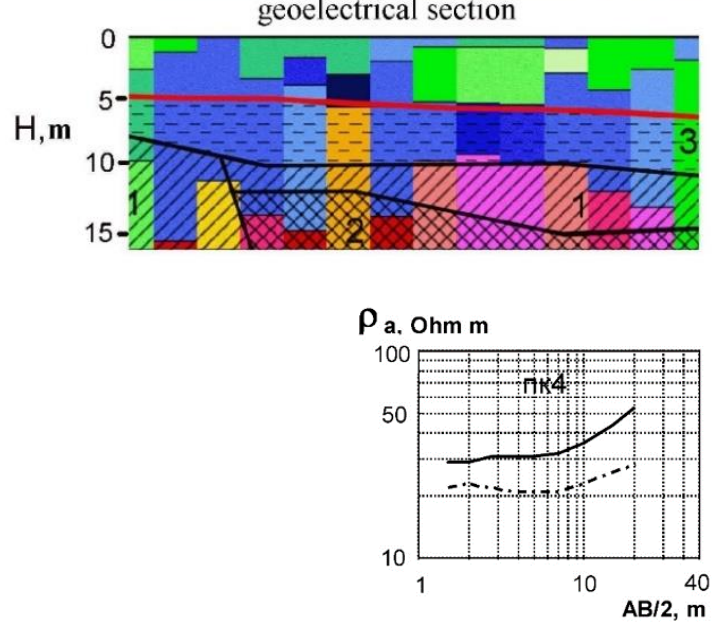

Структура разрезов кажущихся сопротивлений для применяемых методов зондирований схожа (рис. 4). Но, тем не менее, имеются отличия. Кривые зондирований в проводящей зоне (ПК 3-9), полученные комбинированной установкой, быстрее выходят на асимптоту подстилающего слоя (рис. 4 с) и аномальная область ограничивается разносом $\mathrm{AM}=10$ м (рис. $4 \mathrm{a}, \mathrm{c}$ ). На пикете 4 аномалия от симметричной установки вытянута вглубь разреза, а в комбинированной нижняя граница аномалии локализуется на малых разносах, что отразилось в разной вертикальной мощности проводящего слоя, полученной при интерпретации. На геоэлектрических разрезах также наблюдаются различия. В основном они касаются центральной части. На этом участке исследований по результатам интерпретации значения сопротивления грунта, полученные комбинированными зондированиями, выше (50-70 Ом·м), что указывает на более устойчивое состояние откоса плотины. Проводящий слой пониженных удельных сопротивлений смещен на уровень аллювиальных отложений, подстилающих основание плотины. На разрезе вертикальных электрических зондирований на пикетах 4, 5 в теле плотины наблюдаются небольшие проводящие зоны $\rho=15$ Ом·м. (рис. 4 b). На обоих разрезах на пикетах 7 и 10 выявлены области наиболее пониженных значений сопротивлений $\rho=15-$ 18 Ом·м в основании плотины, что указывает на повышенную фильтрацию воды из водоема в этих местах.

\section{b}

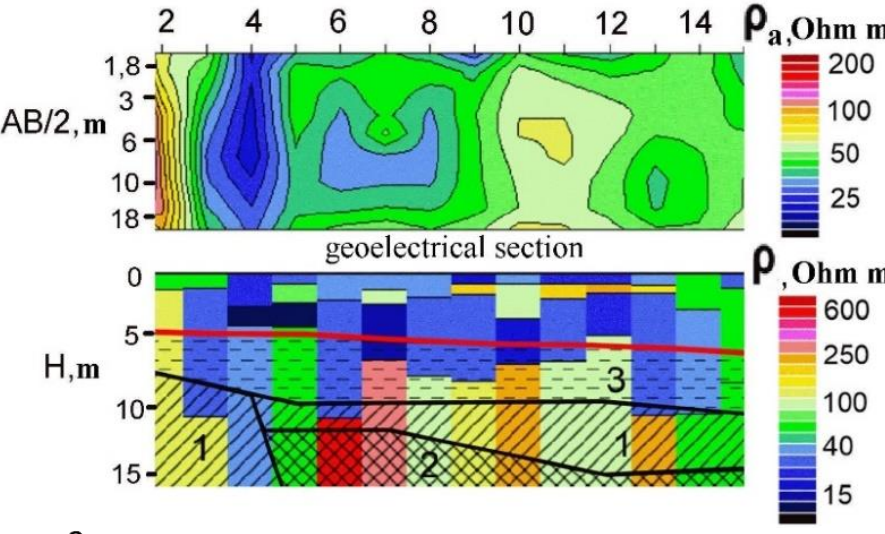

C

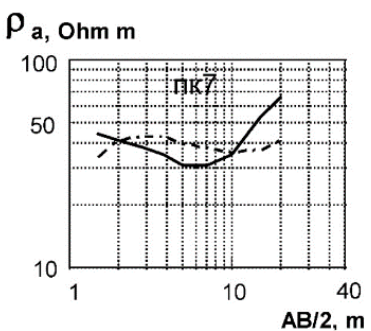

Рис. 4. Результаты исследований на грунтовой плотине. Разрезы кажущихся сопротивлений ( $\left.\rho_{\kappa}\right)$ и геоэлектрические: $a-$ установка AMN+NMA; $b$ - установка AMNB (S). Условные обозначения для геологической основы: 1 - элювиальные суглинки; 2 порфириты; 3 - аллювиальные отложения. Красная линия - основание плотины. Кривые электрических зондирований на пикетах 4 и 7 от установок AMN+NMA (сплошная линия) и $\mathrm{AMNB}(S)$ (пунктирная линия) $-c$.

[Fig. 4. Results of the research at the groundwater dam. The sections of apparent resistivity $\left(\rho_{\mathrm{K}}\right)$ and geoelectrical arrays: $(a)-$ AMN+NMA array; (b) - AMNB (S) array. Legend for the geological basis: (1) eluvial loams; (2) porphyrites; (3) alluvial deposits. Red line is the base of the dam. The electrical sounding curves at pickets 4 and 7 from the AMN + NMA (solid line) and the AMNB (S) (dotted line) arrays (c).] 
Экспериментальные работы с комбинированной методикой зондирований выполнили на Шиловском медно-скарновом золотосодержащем месторождении, расположенном в 25 км к западу от г. Екатеринбурга. Медно-магнетитовые руды практически отработаны шахтным способом. Медно-сульфидная минерализация фиксируется в тектонических нарушениях, локализуясь как в скарнах, так и в габбро, а также в диоритах, в зонах развития кварц-серицитовых метосамотитов. Рудная минеральная ассоциация представлена халькопиритом, пиритом, борнитом, магнетитом и др. Профиль исследований проходил по гранодиоритам и пересекал контакт гранат-пироксеновых скарнов с магнетитовой и сульфидной минерализацией с гранодиоритами в районе 12 пикета. Точки зондирований располагались через 20 м. Полуразносы для установок составляли: AMNB (S) - 1.5; 2, 2.8; 3.8; $5 ; 7 ; 10 ; 15 ; 20$; $28 ; 38 ; 50 ; 70 ; 100 \mathrm{~m} ; \mathrm{AMN}+\mathrm{NMA}-2.25 ; 3 ; 4.2 ; 5.7 ; 7.5$; $10.5 ; 15 ; 22.5 ; 30 ; 42 ; 57 ; 75 ; 105 ; 150$ м. Разрезы кажущихся сопротивлений построены от эффективной глубины $\mathrm{h}_{\text {эф}}$, предусмотренной программой IPI 2win. При одинаковом положении крайних заземлений $\mathrm{h}_{\text {эф }}$ больше в комбинированной установке.

Результаты зондирований с комбинированной и

a

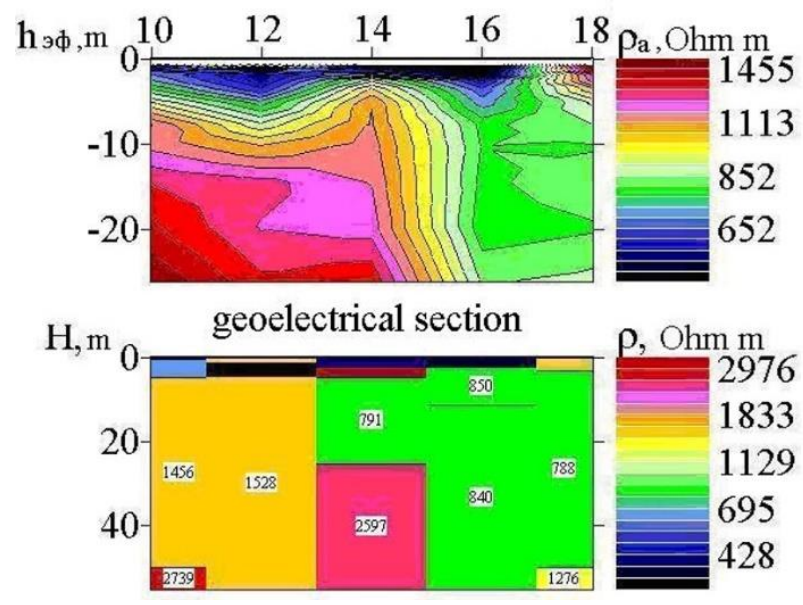

P a, Ohm m

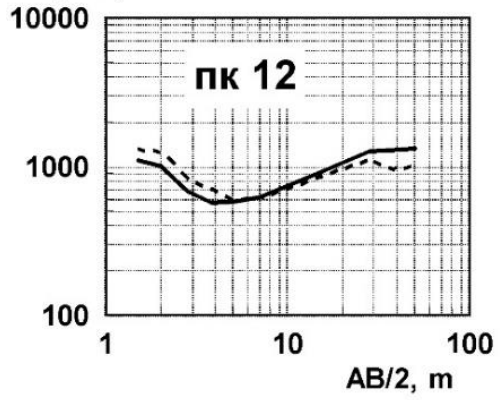

симметричной установками имеют отличия (рис. 5). На разрезе $\rho_{\kappa}$ от установки AMN+NMA на ПК 10-14 ниже $\mathrm{h}_{\ni ф}=10$ м средние значения кажущихся сопротивлений составляют 1200-1300 Ом•м, а от установки AMNB (S) - 950-1000 Ом·м (рис. 5 a, b). На пикете 14 вертикальными электрическими зондированиями (AMNB (S)) выявлена аномалия пониженных значений $\rho_{\mathrm{\kappa}}$, тянущаяся от поверхности на глубину. На разрезе комбинированных зондирований это аномалия отсутствует. Различия полученных данных происходит, по всей видимости, за счет разного влияния краевых эффектов от геологических неоднородностей среды, в первую очередь приповерхностных. Результаты инверсии также отличаются. Контакт скарнов с гранодиоритами на пикетах 10-12 в комбинированной установке выделяется удельным сопротивлением 1500 Ом·м. В симметричной установке на ПК 12 сопротивление понижается до 800 Ом·м, а на ПК14-18 на глубине 5-10 м прослеживается проводящий слой (300-600 Ом·м). На разрезе комбинированных зондирований проводящая зона с пикета 14 имеет большую вертикальную мощность и сопротивление 800 Ом·м. Надо отметить, что по результатам интерпретации вертикальных зондирований на разрезе ниже 10 м сопротивление среды

b

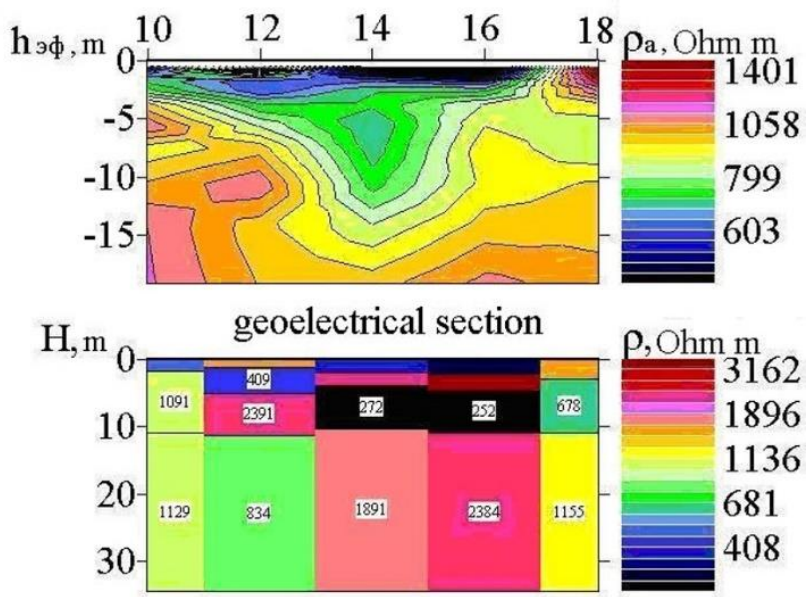

C

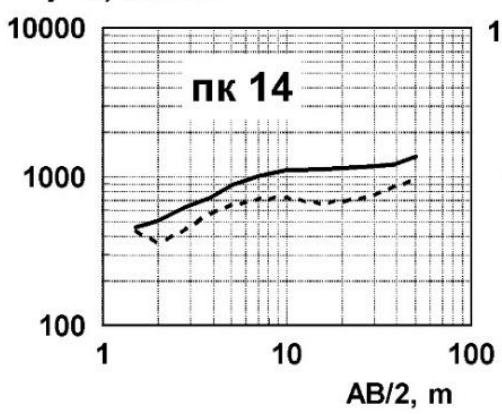

Рис. 5. Результаты исследований на Шиловском месторождении. Разрезы кажущихся сопротивлений ( $\left.\rho_{\kappa}\right)$ и геоэлектрические: $a$ - установка AMN+NMA; $b$ - установка $\mathrm{AMNB}(\mathrm{S})$. Кривые электрических зондирований на пикетах 12, 14 и 16 от установок $\mathrm{AMN}+\mathrm{NMA}$ (сплошная линия) и $\mathrm{AMNB}(S)$ (пунктирная линия) $-c$.

[Fig. 5. Results of the research at the Shilovo deposit. The sections of apparent resistivity $\left(\rho_{\mathrm{\kappa}}\right)$ and geoelectrical arrays: $(a)-\mathrm{AMN}+\mathrm{NMA}$ array; $(b)$ - AMNB (S) array. The electrical sounding curves at pickets 12, 14, and 16 from the AMN + NMA (solid line) and the AMNB (S) (dotted line) arrays $(c)$.] 
по горизонтали меняется от пикета к пикету. Скорее всего, это связано с погрешностью инверсии за счет неуверенного выхода кривой зондирования на асимптоту нижнего слоя. Например, на пикете 16 комбинированной установкой получена двухслойная кривая зондирования, а симметричной установкой - четырехслойная со слабым выходом асимптоты на последний слой (рис. 5 с).

\section{Обсуждение результатов}

Математическое моделирование электрических зондирований с комбинированной установкой AMN+NMA над локальными неоднородностями было выполнено с целью определения влияния неоднородностей на электрическое поле в точке измерения, выражающееся краевыми эффектами на разрезах кажущихся сопротивлений. Предложенная методика зондирований отличается от применяемых методов сопротивлений тем, что в приемной линии один электрод (M), находящийся в центре установки, остается неподвижным, а второй перемещается, и измерения проводятся с обеих сторон от него. Вычисляется среднее значение сопротивления, которое относится к центральному электроду. При такой методике измерений уменьшается влияние неоднородностей, находящихся во вмещающей среде, на электрическое поле вблизи приемной линии (MN), когда ее размер становится больше глубины залегания объекта, или приемный электрод выходит за границы находящегося на поверхности объекта. В качестве модели рассмотрели полушар, аппроксимирующий, например, кору выветривания в рыхлых осадочных породах. Шар послужил моделью погруженной геоэлектрической неоднородности. Для сравнения рассчитали разрезы кажущихся сопротивлений над этими же моделями при вертикальных электрических зондированиях с установкой $\mathrm{AMNB}(\mathrm{S})$, широко применяемых в электроразведке.

Расчеты провели для хорошо проводящих объектов $\rho_{2} / \rho_{1}=0.1$ и слабо проводящих $\rho_{2} / \rho_{1}=10$. В этом случае наиболее максимально проявляются краевые эффекты. По разрезам кажущихся сопротивлений можно сделать следующие выводы. Геоэлектрические неоднородности с малой вертикальной мощностью, выходящие на поверхность (модель полушар) меньше создают краевых эффектов в установке AMN+NMA, а над непроводящим объектом они практически отсутствуют. Нижняя граница неоднородности четко выделяется. На разрезах от установки AMNB (S) горизонтальные размеры объекта уверенно определяются, а вертикальная мощность в несколько раз увеличена за счет ложного аномального эффекта. Это же наблюдается на разрезах кажущихся сопротивлений над шаром. Погруженная локальная неоднородность хорошо оконтуривается комбинированным методом. В методе вертикальных электрических зондирований нижняя граница шара не определяется, так как аномалия вытянута вглубь разреза.
Экспериментальные примеры показали, что отличие в структуре разрезов кажущихся сопротивлений для рассматриваемых установок зависит от геологических особенностей среды. Над горизонтально-слоистой средой в песчано-глинистых и осадочных породах, имеющих низкие удельные сопротивления, как видно по первому эксперименту, кривые зондирования могут иметь различия, но разрезы близки по структуре. Поскольку для установки AMN+NMA не разработана программа 2-D инверсии, количественную интерпретацию полученных данных выполнили по программе 1D инверсии, чтобы можно было сравнить геоэлектрические разрезы от двух установок. Результаты инверсии для горизонтально-слоистой среды отличаются в основном разной вертикальной мощностью проводящих и непроводящих участков, зависящей от того, что кривые зондирования в комбинированной установке быстрее выходят на асимптоту нижнего слоя. Над коренными породами с большим удельным сопротивлением, особенно в контактных и переходных зонах разных пород, кривые зондирования могут значительно отличаться, как это наблюдалось во втором эксперименте. Поэтому здесь на некоторых участках результаты инверсии могут иметь большие различия.

Таким образом, по результатам моделирования установлено, что комбинированная установка AMN+NMA более четко выделяет близповерхностные и погруженные локальные геологические неоднородности, а краевые эффекты во вмещающей среде менее выражены по сравнению с симметричной установкой $\mathrm{AMNB}(\mathrm{S})$. Экспериментальные исследования в некоторых случаях подтверждают установленные моделированием преимущества комбинированной методики зондирований.

\section{Заключение}

Выполнено математическое моделирование электрических зондирований с комбинированной $\mathrm{AMN+NMA} \mathrm{и} \mathrm{симметричной} \mathrm{AMNB}(\mathrm{S})$ установками над локальными неоднородностями. По теоретическим разрезам кажущихся сопротивлений над шаром и полушаром, аппроксимирующими геологические неоднородности, определено, что приповерхностные и погруженные объекты четко выделяются комбинированной установкой с минимальным проявлением краевых эффектов. Проведены натурные эксперименты, которые показали следующее: глубина исследований при одинаковых положениях крайних заземлений больше в установке AMN+NMA; блочное строение среды и приповерхностные неоднородности приводят к структурным различиям геоэлектрических разрезов в рассматриваемых установках. С учетом преимуществ, установленных теоретическими и экспериментальными исследованиями, комбинированная установка может использоваться для электрических зондирований верхней части геологического разреза, а также в электротомографии горных пород. 
Конфликт интересов: Автор декларирует отсутствие явных и потенциальных конфликтов интересов, связанных с публикацией настоящей статьи.

\section{ЛИТЕРАТУРА}

1. Loke M. H., Barker R. D. Rapid least-squares inversion of apparent resistivity pseudosection by a quasi-Newton method // Geoelectrical Prospecting. 1996. V. 44. P. 131-152. DOI: https://doi.org/10.1111/j.1365-2478.1996.tb00142.x

2. Santos F. A., Sultan A. S. On the 3-D inversion vertical of vertical electrical soundings: application to the South Ismailia area - Cairo Desert Roud, Cairo, Egypt // Journal of Applied Ge ophysics. 2008. V. $65 . \quad$ P. 97-110. DOI: https://doi.org/10.1016/j.jappgeo.2008.06.001

3. Shima H. The effects on reconstructed images surrounding resistivity structures in resistivity tomography. Report, presented at 59 th Annual International SEG Meeting. Dallas, 1989.

4. Loke M. H. Tutorial: 2-D and 3-D electrical imaging surveys. [Электронный ресурc] 2009. URL: https://www.geotomosoft.com/coursenotes.zip (дата обращения: 27.05.2021)

5. Бобачев А. А., Марченко М. Н., Модин И. Н., Перваго Е. В., Урусова А. В., Шевнин В. А. Новые подходы к электрическим зондированиям горизонтально-неоднородных сред // Физика Земли. 1995. № 12. С. 79-90.
6. Шестаков А. Ф., Федорова О. И. Способ геоэлектроразведки. Патент №2581768. заявка №2014147521/28 от 25.11.2014. опубликовано 20.04.2016. Бюл. №11.

7. Федорова О. И. Электрические зондирования с комбинированной трехэлектродной установкой AMN+NMA // Известия вузов. Горный журнал. 2018. №1. С. 115-119. DOI: https://doi.org/10.25698/UGV.2020.3.5.37

8. Федорова О. И. Математическое моделирование электрических зондирований над вертикальным пластом с комбинированной и симметричной установками // Уральский геофизический вестник. 2020. №3. С. 37-43. DOI: https://doi.org/10.21440/0536-1028-2018-1-115-119

9. Olga I. F., Gorshkov V. Y. New technique of electrical soundings: theoretical modeling and experimental application in study of state of the soil dam // Acta Geodaetica et Geophysica. 2020. № 55. P.1-9. DOI: https://doi.org/10.1007/s40328-019-00277-1 10. Kenneth L. Cook, Robert G. Van Nostrand Interpretation of resistivity data over filled sinks // Geophysics. 1954. Vol. XIX. № 4. Р. 761-70.

11. Заборовский А. И. Электроразведка. М.: Гостоптехиздат, 1943. $444 \mathrm{c}$.

12. Bobachev A. A. IPI2Win(MT) v2.0 User's Guide: PDF document. [Электронный ресурс] Moscow State University. 2002. URL: http://geoph ys.geol.msu.ru/ipi_mts/wmts_en.pdf (дата обращения 627.05 .2021

UDC 550.837

DOI: https://doi.org/10.17308/geology.2021.3/3653

ISSN 1609-0691

Received: 27.05.2021

Accepted: 02.09.2021

Published online: 07.10.2021

\title{
Electrical soundings with combined and symmetrical arrays over geological inhomogeneities: mathematical modelling and experimental examples
}

\author{
C 2021 O. I. Fedorova ${ }^{\bowtie}$
}

Institute of Geophysics UB of RAS, 100 Amundsena ul,. Ekaterinburg 620016, Russian Federation

\begin{abstract}
Introduction: Electric soundings with direct current are widely used for the solution of geological and geotechnical problems by using four-electrode symmetric AMNB arrays, Schlumberger (S) and Wenner (V), which allows reliably determining the thickness and resistivity of layers for horizontally layered media. Geological inhomogeneities that are present in the media create edge effects which are reflected on the sections of apparent resistivity as additional anomalous areas and influence the accuracy of quantitative interpretation. To reduce edge effects, we suggested a method of electric soundings with the AMN+NMA $\mathrm{B} \rightarrow \infty$ combined array that conducts double calculations in relation to the central receiving electrode (M). Methodology: We conducted mathematical modelling of soundings with the AMNB (S) and AMN + NMA arrays over local objects, a sphere and a hemisphere, approximating geoelectrical inhomogeneities. Experimental research was conducted on two sites using a combined and, for comparison, a symmetric array.
\end{abstract}

The content is available under Creative Commons Attribution 4.0 License.

\footnotetext{
Olga I. Fedorova, e-mail: fougeo@mail.ru
} 
Results: The calculated theoretical sections of apparent resistivity showed that the subsurface and immersed local inhomogeneities were distinctly contoured by the combined array with minimal display of edge effects. There were edge effects on the sections obtained using the symmetric array, and the lower border was poorly determined. Experimental examples found differences in the structure of the sections of apparent resistivity for the considered arrays, which depended on the geological features of the medium. The sections are similar in structure over the medium with horizontal stratification. The results of inversion mainly differed in the vertical thickness of layers. The sounding curves and the results of inversion may vary considerably over the bedrocks with great resistivity in the near-contact zones.

Conclusions: Based on the conducted modelling and experiments, it can be concluded that the combined AMN+NMA array has some advantages as compared to the widely used AMNB array and can be used for electrical sounding of the upper part of the geological section as well as in electrical resistivity tomography of rocks.

Keywords: Electrical sounding, combined array, symmetric array, section of apparent resistivity, geoelectric inhomogeneity, edge effects.

For citation: Fedorova O. I. Electrical soundings with combined and symmetrical arrays over geological inhomogeneities: mathematical modelling and experimental examples. Vestnik Voronezhskogo gosudarstvennogo universiteta. Seriya: Geologiya - Proceedings of Voronezh State University. Series: Geology, 2021, no. 3, pp. 87-95. DOI: https://doi.org/10.17308/geology.2021.3/3653

Conflict of interests: The authors declare the absence of obvious and potential conflicts of interest related to the publication of this article.

\section{REFERENCES}

1. Loke M. H., Barker R. D. Rapid least-squares inversion of apparent resistivity pseudosection by a quasi-Newton method. Geoelectrical Prospecting, 1996, vol. 44, pp. 131-152. DOI: https://doi.org/10.1111/j.1365-2478.1996.tb00142.x

2. Santos F. A., Sultan A. S. On the 3-D inversion vertical of vertical electrical soundings: application to the South Ismailia area - Cairo Desert Roud, Cairo, Egypt. Journal of Applied Geophysics, 2008, vol. 65, pp. 97-110. DOI:

https://doi.org/10.1016/j.jappgeo.2008.06.001

3. Shima $\mathrm{H}$. The effects on reconstructed images surrounding resistivity structures in resistivity tomography. Report, presented at 59 th Annual International SEG Meeting, Dallas, 1989. 4. Loke M. H. Tutorial: 2-D and 3-D electrical imaging surveys. 2009, Available at: https://www.geotomosoft.com/coursenotes.zip 5. Bobache A. A., Marchenko M. N., Modin I. N., Pervago E. V., Urusova A. V., Shevnin V. A. Novyye podkhody k elektricheskim zondirovaniyam gorizontal'no- neodnorodnykh sred [New approaches to electrical soundings of horizontally inhomogeneous media]. Fizika Zemli - Physics of the Solid Earth, 1996, no. 12, pp. 1075-1086. (In Russ)

6. Shestakov A. F. Fedorova O. I., Sposob geoelektrorazvedki [Method of geoelectrical exploration]. Patent $R F$ no. 2581768, Declared no. 2014147521/28 25.11.2014, Published 20.04.2016, Bulletin no. 11. (In Russ)
7. Fedorova O. I. Elektricheskiye zondirovaniya s kombinirovannoy trekhelektrodnoy ustanovkoy AMN+NMA [Electrical sounding with the combined three-electrode AMN + NMA array]. Izvestiya vuzov. Gornyi zhurnal - Izvestiya vuzov. Mining Journal, 2018, no. 1, pp. 115-119. DOI: https://doi.org/10.21440/0536-1028-2018-1-115-119. (In Russ)

8. Fedorova O. I. Matematicheskoye modelirovaniye elektricheskikh zondirovaniy nad vertikal'nym plastom s kombinirovannoy i simmetrichnoy ustanovkami [Mathematical modeling of electrical sounding over vertical layer with combined and symmetric arrays]. Ural'skiy geofizicheskiy vestnik - Ural Geophysical Bulletin, 2020, no. 3, pp. 37-43. DOI: https://doi.org/10.25698/UGV.2020.3.5.37. (In Russ)

9. Olga Ivanovna Fedorova, Vitaliy Yurievich Gorshkov New technique of electrical soundings: theoretical modeling and experimental application in study of state of the soil dam. Acta Geodaetica et Geophysica, 2020, no. 55, pp. 1-9. DOI: https://doi.org/10.1007/s40328-019-00277-1

10. Kenneth L. Cook, Robert G. Van Nostrand Interpretation of resistivity data over filled sinks. Geophysics, 1954, vol. XIX, no. 4, pp. 761-70.

11. Zaborovsky A.I. Elektrorazvedka [Electrical prospecting]. Moscow, Gostoptekhizdat publ., 1943, 444 p. (In Russ)

12. Bobachev A. A. IPI2Win(MT) v2.0 User's Guide. Moscow State University, 2002, Available at: http://geoph ys.geol.msu.ru/ipi_mts/wmts_en.pdf (In Russ)
Федорова Ольга Ивановна - к. г.-м. н., с. н. с., Институт геофизики им. Ю. П. Булашевича УрО РАН, Екатеринбург, Российская Федерация; E-mail: fougeo@ mail.ru; ORCID: http://orcid.org/0000-0001-9882-8313

Автор прочитал и одобрил окончательный вариант рукописи.
Olga I. Fedorova - PhD in Geol-Min., senior researcher, Institute of Geophysics UB of RAS, Russian Federation; E-mail: fougeo@mail.ru; ORCID: http://orcid.org/0000-0001-98828313

Author has read and approved the final manuscript. 